\title{
THE NON-UNIFORM MOTION OF A THIN SMOOTH RIGID INCLUSION THROUGH AN ELASTIC SOLID*
}

\author{
BY \\ L. M. BROCK \\ University of Kentucky
}

\begin{abstract}
A two-dimensional example of non-uniform motion by a smooth, relatively thin, symmetric rigid inclusion through an elastic solid is analyzed. Integral solutions to the related boundary-initial value problem are obtained for the case of edge speeds bounded by the dilatational wave speed at large times. The wavefront patterns, including the possibility of Mach waves, are noted. Some attention is focused on the case of the straight wedge, and logarithmic singularities are found at its edge for subsonic speeds.
\end{abstract}

1. Introduction. The study of rigid inclusion motion through an elastic solid has implications in the understanding of material cutting and internal failure of reinforced material. In this article an idealized two-dimensional example of such motion is analyzed. A smooth, relatively thin, symmetric rigid inclusion moves along its symmetry plane from a position of static equilibrium through an isotropic, homogeneous, linearly elastic solid. The inclusion speed is non-uniform and largely arbitrary. The inclusion profile is also largely arbitrary but for illustration much of the analysis treats the straight wedge. Attention is focused on the leading edge of the inclusion. Thus, during the motion the analysis considers a semi-infinite inclusion in an unbounded solid.

The static equilibrium position (time $t<0$ ) is shown in Fig. 1. For convenience the variable $s=c_{1} t$ is introduced. Here $c_{1}, c_{2}$ and $\mu$ are the dilatational and rotational wave speeds and the shear modulus in the solid. The profile function $f(\xi) \geq 0, \xi \geq 0$ is continuous, smooth and finite for finite $\xi$ with $f(0)=0$. At $t=0$ the inclusion undergoes the rigid body translation $h(s) \geq 0, s \geq 0$ in the positive $x$-direction where $h(s)$ is continuous, at least piecewise smooth and finite for finite $s$, with $\dot{h}(s) \geq 0$ and $h(0)=0$. Here ()$^{\prime} \equiv d() / d \xi$ and $\left(^{\circ}\right) \equiv d() / d s$. The solid is assumed to remain in frictionless contact with the moving inclusion.

Because the wave motion is of chief interest, the superposition-related problem obtained by subtracting the static field from the complete solution is studied. The initial conditions on this problem are

$$
s \leq 0: u, v \equiv 0
$$

where $u, v$ are the $x, y$-displacements. Attention is confined to the region $y<0$ by appending the boundary conditions

$$
v=f(-x) H(-x)-f[h(s)-x] H[h(s)-x], \quad \sigma_{x y}=0
$$

\footnotetext{
* Received September 1, 1977; revised version reviewed January 11, 1978.
} 


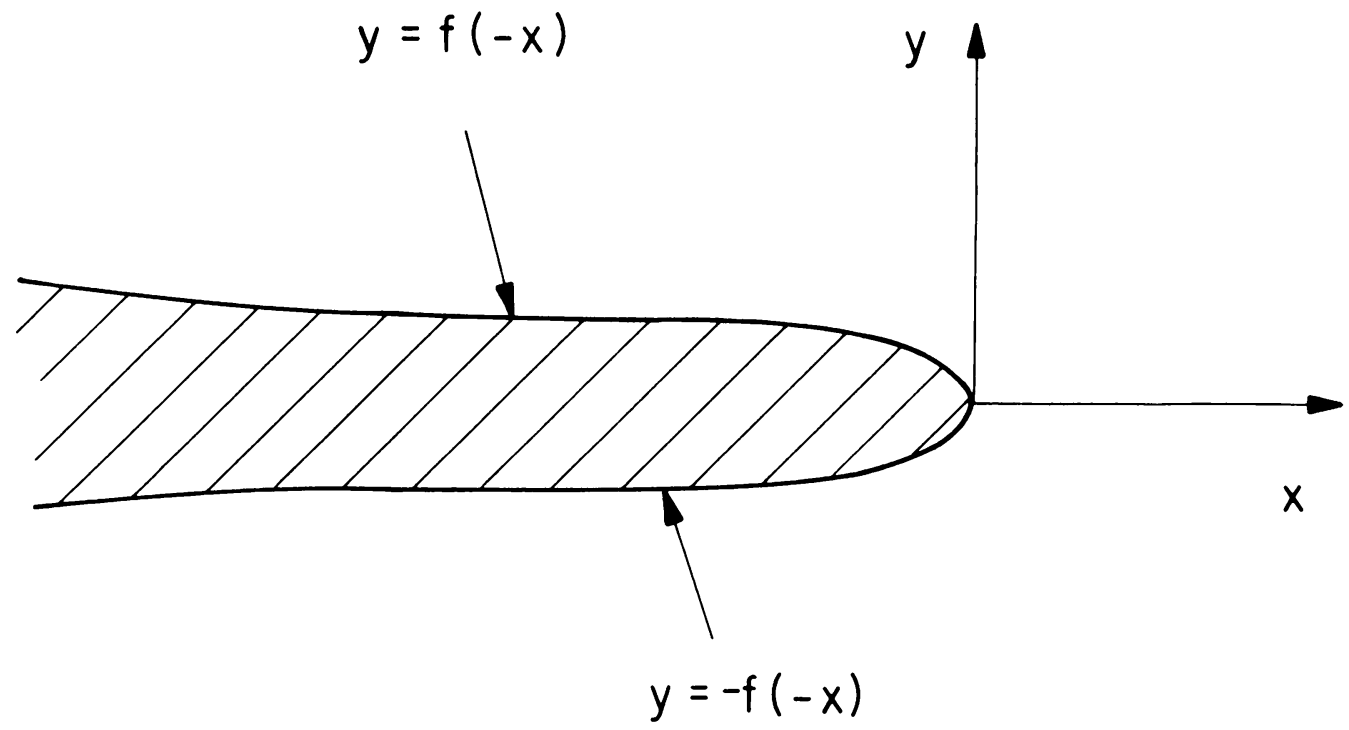

FIG. 1. Initial inclusion geometry.

along the symmetry plane $y=0$, where $(1.2 a)$ is an approximation for small displacements [1] and $H()$ is the Heaviside step function. The first term in (1.2a) removes the normal displacement existing prior to $s=0$ while the second term imposes the normal displacement due to the traveling inclusion for $s \geq 0$. The governing differential equations for $y<0$ are

$$
\sigma_{x, x}+\sigma_{x y, y}=\mu \ddot{u} / m^{2}, \quad \sigma_{x y, x}+\sigma_{y, y}=\mu \ddot{v} / m^{2}
$$

where ( $)_{a} \equiv \partial() / \partial a$ and $m=c_{2} / c_{1}(0<m<1 / \sqrt{ } 2)$. The constitutive equations are

$$
\frac{m^{2}}{\mu} \sigma_{x}=u_{, x}+\left(1-2 m^{2}\right) v,{ }_{, y}, \quad \frac{m^{2}}{\mu} \sigma_{y}=v_{, y}+\left(1-2 m^{2}\right) u_{, x}, \quad \frac{1}{\mu} \sigma_{x y}=u_{, y}+v_{, x} .
$$

In addition the solutions should be bounded above in $y<0$ for finite $s$.

2. The transform solutions. The one-sided and bilateral Laplace transforms

$$
\hat{g}(p)=\int_{0}^{\infty} g(s) \exp (-p s) d s, \quad g^{*}(p, q)=\int_{-\infty}^{\infty} g(x) \exp (-p q x) d x
$$

over $s$ and $x$ are introduced. Here $p$ is real and positive and large enough to insure convergence of (2.1a) while $q$ is in general complex. Operating on (1.3) with (2.1) in view of (1.1) and (1.4) yields the transformed equations for $y<0$ :

$$
\begin{array}{cccc}
m^{2} \hat{u}^{*}{ }_{y y}-p^{2} a^{2} \hat{u}^{*}+p q\left(1-m^{2}\right) \hat{v}^{*},{ }_{y}=0, & a=\left(1-q^{2}\right)^{1 / 2}, & \operatorname{Re}(a) \geq 0, & (2.2 a, b) \\
\hat{v}^{*},{ }_{y y}-m^{2} p^{2} b^{2} \hat{v}^{*}+p q\left(1-m^{2}\right) \hat{u}^{*},{ }_{y}=0, & b=\left(m^{-2}-q^{2}\right)^{1 / 2}, & \operatorname{Re}(b) \geq 0 . & (2.2 c, d)
\end{array}
$$

Here $a, b$ are defined in the plane cut along $\operatorname{Im}(q)=0,|\operatorname{Re}(q)|>1$ and $|\operatorname{Re}(q)|>1 / m$, respectively. Operating on (1.2) with (2.1) in view of (1.1) and (1.4) and recognizing that 
$f(0)=0$ yields the transformed conditions along $y=0$ :

$$
\begin{gathered}
\hat{v}^{*}=V(p, q), \quad \hat{\sigma}_{x y}{ }^{*}=0 \\
p q V(p, q)=\int_{0}^{\infty} \exp (p q \xi) f^{\prime}(\xi)\left[-\frac{1}{p}+\int_{0}^{\infty} \exp (-p[q h(\tau)+\tau]) d \tau\right] d \xi .
\end{gathered}
$$

The double transform $V$ exists if $\operatorname{Im}(p)=0, \operatorname{Re}(p)>0$ and $\operatorname{Re}(q)<0$ while $\operatorname{Re}(q)>-\tau /$ $h(\tau), \tau \rightarrow \infty$. Thus, to insure that the convergence strip for $V$ in the $q$-plane does not vanish, $h(s)$ should behave no worse than $0(s), s \rightarrow \infty$. Thus, the edge speed is bounded by the dilatational wave speed at infinitely large times after motion begins. A set of solutions to (2.2) and (2.3) which are bounded for $y<0$ is easily obtained as

$$
\begin{aligned}
\hat{u}^{*} & =A \exp (p a y)+B \exp (p b y), \hat{v}^{*}=\frac{a}{q} A \exp (p a y)-\frac{q}{b} B \exp (p b y), \\
\frac{1}{\mu} \hat{\sigma}_{x}^{*} & =\frac{p}{q}\left(m^{-2}-2 a^{2}\right) A \exp (p a y)+2 p q B \exp (p b y), \\
\frac{1}{\mu} \hat{\sigma}_{y}^{*} & =\frac{p}{q}\left(b^{2}-q^{2}\right) A \exp (p a y)-2 p q B \exp (p b y), \\
\frac{1}{\mu} \hat{\sigma}_{x y}{ }^{*} & =2 p a A \exp (p a y)+\frac{p}{b}\left(b^{2}-q^{2}\right) B \exp (p b y), \\
A & =\frac{m^{2} q}{a}\left(b^{2}-q^{2}\right) V(p, q), \quad B=-2 m^{2} q b V(p, q) .
\end{aligned}
$$

3. Transform inversions. The displacements $u, v$ are obtained. Although some features differ, the inversions of the stress transforms (2.5)-(2.7) follow the same pattern. The inverse bilateral transform is defined as

$$
g(x)=\frac{p}{2 \pi i} \int_{a_{0^{-}-i \infty}}^{a_{0}+i \infty} g^{*}(p, q) \exp (p q x) d q
$$

where the real constant $q_{0}$ is chosen so that the integration path lies in the region of analyticity for $g^{*}$ and convergence of $(2.1 b)$. Substitution of (2.4a) and (2.8) into (3.1) yields the one-sided Laplace transform

$$
\hat{u}=\frac{m^{2} p}{2 \pi i} \int_{-i \infty}^{i \infty}\left[\frac{b^{2}-q^{2}}{a} \exp (p a y)-2 b \exp (p b y)\right] q V(p, q) \exp (p q x) d q
$$

where in view of $(2.3 c)$ we can choose $q_{0}=0$. Upon substitution of $(2.3 c)$ and interchanging the order of integration, the first term in (3.2) becomes

$$
\begin{gathered}
\frac{m^{2}}{2 \pi i} \int_{0}^{\infty} \int_{0}^{\infty} f^{\prime}(\xi) \int_{-i \infty}^{i \infty} \frac{b^{2}-q^{2}}{a} \exp (p(a y+q x+q \xi)-p[q h(\tau)+\tau]) d q d \xi d \tau \\
-\frac{m^{2}}{2 \pi i} \int_{0}^{\infty} f^{\prime}(\xi) \int_{-i \infty}^{i \infty} \frac{b^{2}-q^{2}}{a p} \exp (p(a y+q x+q \xi)) d q d \xi .
\end{gathered}
$$

The Cagniard-deHoop [2] scheme is followed to allow inversion of (3.3) by inspection. Contours in the $q$-plane are sought along which the exponentials involving $q$ in the first and second terms in (3.3) assume the form $\exp (-p n)$, where $n$ is real and positive. Such 
contours are readily found to be defined by $q_{1}^{ \pm}(n, \tau)$ and $q_{1}^{ \pm}(n, 0)$, respectively:

$$
\begin{array}{r}
\rho^{2} q_{1}^{ \pm}(n, \tau)=n \zeta \pm i|y|\left(n^{2}-\rho^{2}\right)^{1 / 2}, \quad \rho(\tau)=\left(y^{2}+\zeta^{2}\right)^{1 / 2}, \\
\zeta(\tau)=h(\tau)-x-\xi .
\end{array}
$$

This equation defines the branch of a hyperbola with vertex $q_{1}{ }^{ \pm}=\zeta / \rho$ and asymptotes $\arg \left(q_{1}^{ \pm}\right)= \pm \tan ^{-1}(|y| / \zeta)$. Thus the hyperbola does not intersect the branch cuts for $a$ and the integrands of (3.3) are analytic in the region between the hyperbola and the $\operatorname{Im}(q)$-axis. The hyperbola lies in the half-plane where $\operatorname{Re}(q)$ has the same sign as $\zeta$ and the exponentials involving $q$ in (3.3) vanish in this half-plane when $|q| \rightarrow \infty$ for positive real $p$. Thus, the Cauchy theorem can be used to change the integration path in (3.3) to the contours $q_{1}{ }^{ \pm}$where integration is now with respect to $n$. As these contours are traversed from $\infty$ in the lower half-plane through the vertex to $\infty$ in the upper half-plane, $n$ varies from $\infty$ to $\rho$ and back to $\infty$. Moreover, the integrands of (3.3) along $q_{1}{ }^{+}$and $q_{1}{ }^{-}$are complex conjugates. In summary (3.3) can be written as

$$
\begin{aligned}
& \frac{m^{2}}{\pi} \int_{0}^{\infty} \int_{0}^{\infty} f^{\prime}(\xi) \int_{\rho(\tau)}^{\infty} Z_{1}\left[q_{1}{ }^{+}(n, \tau)\right] \exp [-p(n+\tau)] d n d \xi d \tau \\
& -\frac{m^{2}}{\pi p} \int_{0}^{\infty} f^{\prime}(\xi) \int_{\rho(0)}^{\infty} Z_{1}\left[q_{1}{ }^{+}(n, 0)\right] \exp (-p n) d n d \xi, \\
& Z_{1}(q)=\operatorname{Im}\left(\frac{b^{2}-q^{2}}{a} \frac{d q}{d n}\right), \quad Z_{2}(q)=\operatorname{Im}\left(b \frac{d q}{d n}\right), \quad Z_{k}(q)=q Z_{k}{ }^{*}(q)
\end{aligned}
$$

The functions of $p$ in the first and second terms of (3.5a) are the one-sided LaPlace transforms of the Dirac delta function $\delta(s-n-\tau)$ and Heaviside step function $H(s-n)$ [3]. A similar procedure can be followed for the second term in (3.2). Then, upon using the sifting property [4] of the delta function to eliminate the $n$-integration, we obtain

$$
\begin{aligned}
u= & \frac{m^{2}}{\pi} \int_{0}^{\infty} \int_{0}^{\infty} f^{\prime}(\xi) Z_{1}\left[q_{1}{ }^{+}(s-\tau, \tau)\right] H[s-\tau-\rho(\tau)] d \xi d \tau \\
& -\frac{2 m^{2}}{\pi} \int_{0}^{\infty} \int_{0}^{\infty} f^{\prime}(\xi) Z_{2}\left[q_{2}{ }^{+}(s-\tau, \tau)\right] H[m(s-\tau)-\rho(\tau)] d \xi d \tau \\
& -\frac{m^{2}}{\pi} \int_{0}^{\infty} f^{\prime}(\xi) \int_{\rho(0)}^{s} Z_{1}\left[q_{1}{ }^{+}(n, 0)\right] d n d \xi \\
& +\frac{2 m^{2}}{\pi} \int_{0}^{\infty} f^{\prime}(\xi) \int_{\rho(0) / m}^{s} Z_{2}\left[q_{2}^{+}(n, 0)\right] d n d \xi, \\
& \rho^{2} q_{2}^{ \pm}(n, \tau)=n \zeta \pm i|y|\left[n^{2}-m^{-2} \rho^{2}\right]^{1 / 2} .
\end{aligned}
$$

By a similar procedure, $v$ follows from (3.6) by replacing $a$ in the first and third terms and $b$ in the second and fourth terms with $q$ while appending the term $f(-x) H(-x) H(s-|y|)$ $-f[h(s-|y|)-x] H[h(s-|y|)-x] H(s-|y|)$. This term arises from the residue of the simple pole in $(2.4 b)$ at $q=0$ which is encountered when the Cauchy theorem is applied to change the integration contours.

4. General wavefront pattern. Some general information about the resulting wavefront pattern in the solid can be obtained from (3.6). The first two terms represent the disturbance generated by the motion of the inclusion for $s \geq 0$. The disturbance due to the 
last two terms is, in effect, a signal that the inclusion accelerates from rest and does not appear instantaneously.

The Heaviside function in the last term in (3.6) confines points $x, y$ within the circle of locus $C=\rho^{2}(0)-m^{2} s^{2}=0$. Solution of the characteristic equations

$$
C=0, \quad d C / d \xi=0
$$

yields the points $x=-\xi,|y|=m s$ on the envelope generated by the circle as $\xi$ varies from 0 to $\infty$. Thus, the disturbance due to the last term occurs in the overlapping rotational plane and cylindrical wave regions $|y| \leq m s(x \leq 0)$ and $r=\left(x^{2}+y^{2}\right)^{1 / 2} \leq m s$.

The Heaviside function in the second term in (3.6) requires that $0 \leq \tau \leq s$ and confines $x, y$ within the circle of locus $C=\rho^{2}(\tau)-m^{2}(s-\tau)^{2}=0$. Solution of (4.1) yields the envelope $x=h(\tau)-\xi,|y|=m(s-\tau)$ as $\xi$ varies from 0 to $\infty$. However, solution of the characteristic equations

$$
C=0, \quad d C / d \tau=0
$$

gives an envelope of the form

$$
x+\xi=h(\tau)+m^{2}(s-\tau) / \dot{h}(\tau), \quad|y|=m(s-\tau)\left[1-m^{2} / \dot{h}^{2}(\tau)\right]^{1 / 2}
$$

which obviously yields real values only if $\dot{h}(\tau)>m$. This result can be visualized by noting that as $\tau$ varies from 0 to $s$ the circle radius vanishes while its center translates. Thus, if $\dot{h}(\tau)<m$ for a given $\tau$, the circle for $\tau+\Delta \tau\left(\Delta \tau \rightarrow 0^{+}\right)$will lie within the circle for $\tau$. These observations indicate that if $\dot{h}(s)>m$, in addition to the wavefronts described for the last term, a rotational $M$ ach wave radiates from the inclusion edge. The wavefronts are parameterized by (4.3) where $\xi=0, s_{1} \leq \tau \leq s$ and are tangent to the cylindrical rotational wavefront $\left[x-h\left(s_{1}\right)\right]^{2}+y^{2}=m^{2}\left(s-s_{1}\right)^{2}$. Here $s_{1}$ is the instant at which $\dot{h}$ exceeds $m$. If at a time $s_{2}>s_{1} \dot{h}$ drops below $m$, the Mach wavefronts separate from the inclusion edge and also become tangent to the cylindrical rotational wavefront $\left[x-h\left(s_{2}\right)\right]^{2}+y^{2}=m^{2}\left(s-s_{2}\right)^{2}$. Eq. (4.3) demonstrates that the Mach wavefronts are straight lines when $\dot{h}=$ constant $>$ $m$.

Eq. (3.6) also indicates that along $y=0$ there are no Rayleigh surface waves and no rotational waves generated by a faster-traveling dilatational wave (head waves). These absences occur because, respectively, the solution transforms have poles only at $q=0$ and the contours $q_{1}^{ \pm}, q_{2}^{ \pm}$do not intersect the branch cuts of the transforms. Both results, in turn, follow from the mixed nature of the boundary conditions (1.2).

A similar discussion applies to the first and third terms in (3.6). The wavefront patterns for two cases of non-constant $\dot{h}$ are shown in Fig. 2. It should be emphasized that the patterns discussed here are general. As will be seen, certain field variables may vanish in various regions behind these wavefronts. Moreover, it is possible to isolate certain interior regions because of interesting solution behavior.

5. Example: the wedge. To illustrate some features of the solution behavior, it is convenient to treat a specific inclusion profile. A simple choice is the straight wedge

$$
f(\xi)=f^{\prime} \xi, \quad f^{\prime}=\text { constant }>0 .
$$

The form of (5.1) allows the field variables to be written in a simpler form than, say, (3.6). For example, the particle velocity $\dot{u}$ is

$$
\dot{u}=-\frac{m^{2}}{\pi} f^{\prime} \int_{0}^{\infty} Z_{1}^{*}\left[q_{1}{ }^{+}(s-\tau, \tau)\right] H[s-\tau-\rho(\tau)] d \tau_{\alpha}
$$



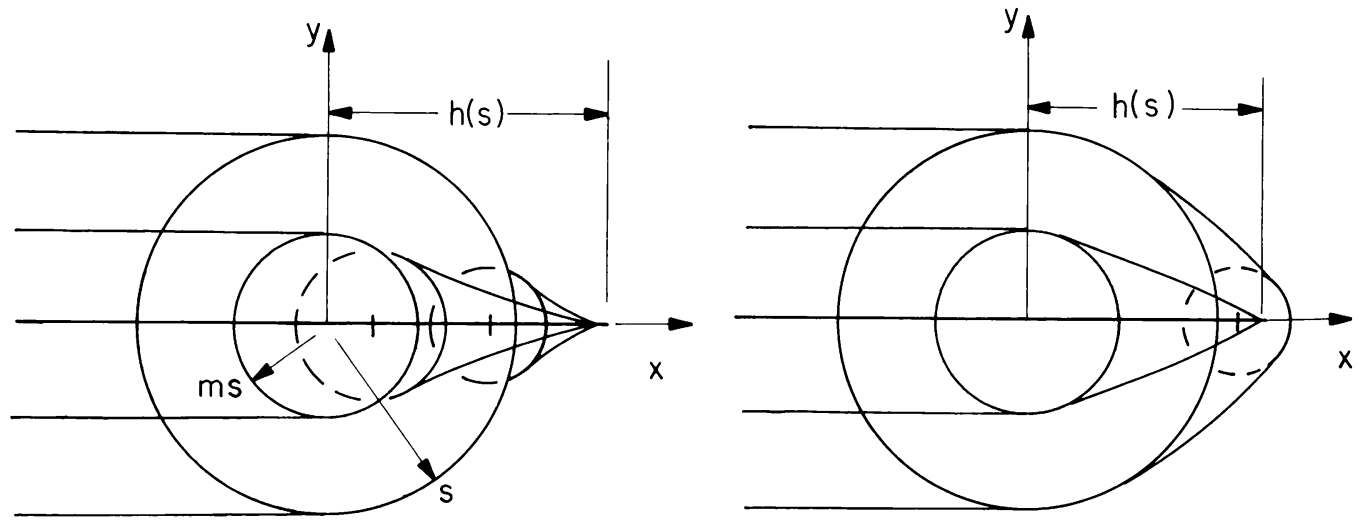

Fig. 2. Two possible wavefront patterns.

$$
\begin{aligned}
& +\frac{2 m^{2}}{\pi} f^{\prime} \int_{0}^{\infty} Z_{2}{ }^{*}\left[q_{2}{ }^{+}(s-\tau, \tau)\right] H[m(s-\tau)-\rho(\tau)] d \tau \\
& +\frac{m^{2}}{\pi} f^{\prime} \int_{r}^{s} Z_{1}{ }^{*}\left[q_{1}{ }^{+}(n, 0)\right] d n-\frac{2 m^{2}}{\pi} f^{\prime} \int_{r / m}^{s} Z_{2}{ }^{*}\left[q_{2}{ }^{+}(n, 0)\right] d n \\
& +f^{\prime} H[h(s-|y|)-x] H(x)-2 m f^{\prime} H[h(s-|y| / m)-x] H(x),
\end{aligned}
$$

where $\rho(\tau)$ is modified by setting $\xi=0$. Eq. (5.2) indicates that $\dot{u}$ does not propagate as a plane wave from the wedge surface. This result is reasonable in view of the frictionless contact. The non-integral terms in (5.2) indicate that a separate class of waves is generated by the wedge edge, whether or not its speed is supersonic.

In the next section the field variable behavior at $y=0, x=h(s)$ is of interest. Therefore, for illustration we present the expression for $\sigma_{y}$ along $y=0$ :

$$
\begin{aligned}
\frac{1}{\mu} \sigma_{y}= & -\frac{m^{2} f^{\prime}}{\pi} \int_{0}^{\infty} \frac{\left(m^{-2}-2 \lambda^{2}\right)^{2}}{\lambda \alpha(1 / \lambda)} \frac{1}{h(\tau)-x} H[s-\tau-|h(\tau)-x|] d \tau \\
& +\frac{4 m^{2} f^{\prime}}{\pi} \int_{0}^{\infty} \frac{\lambda \beta(1 / \lambda)}{h(\tau)-x} H[m(s-\tau)-|h(\tau)-x|] d \tau-\frac{f^{\prime}}{m^{2}} \dot{h}(s) H[h(s)-x] \\
& -\frac{m^{2} f^{\prime}}{\pi} \int_{|x| / s}^{1} \frac{\left(2-w^{2} / m^{2}\right)^{2}}{w^{3} \alpha(w)} d w+\frac{4 m^{2} f^{\prime}}{\pi} \int_{|x| / s}^{m} \frac{\beta(w)}{w^{3}} d w, \\
& \alpha(w)=\left(1-w^{2}\right)^{1 / 2}, \quad \beta(w)=\left(1-w^{2} / m^{2}\right)^{1 / 2}, \quad \lambda=\frac{s-\tau}{h(\tau)-x} .
\end{aligned}
$$

Knowledge of the actual $\tau$-integration limits in (5.3) is required in the next section. The integration paths can be represented as vertical lines in the $x \tau$-plane which are contained within the region bounded by the curves $\tau=0, x_{+}(\tau)$ and $x_{-}(\tau)$, where for the first and second integrals in (5.3),

$$
x_{ \pm}(\tau)=h(\tau) \pm(s-\tau), \quad x_{ \pm}(\tau)=h(\tau) \pm m(s-\tau)
$$

respectively. Two examples involving (5.5b) are shown in Fig. 3. It is seen that $d x_{+} / d \tau<0$ when $\dot{h}(\tau)<m$ and $d x_{+} / d \tau>0$ when $\dot{h}(\tau)>m$. Moreover, the curve $x_{-}$and its counterpart in (5.5a) indicate that $\sigma_{y}=0$ for $y=0, x<-s$. 
6. Behavior at the wedge edge. For illustration, the behavior of the second integral in (5.3) as $x \rightarrow h(s)^{+}$is studied for the case $h(s)<m, h(s)<m s$. We examine a nearby point $x=h(s)+\epsilon$, where $\epsilon$ is positive and vanishingly small. Then, as indicated in Fig. 3, the limits of integration are $0, \tau^{*}<s$. The second integral can be written as

$$
\frac{\hat{\lambda} \beta(1 / \hat{\lambda})}{\dot{h}(\hat{\tau})} \ln \left[\frac{x-h\left(\tau^{*}\right)}{x}\right]+\int_{0}^{\tau^{*}} \frac{\dot{h}(\tau)}{h(\tau)-x}\left[\frac{\lambda \beta(1 / \lambda)}{\dot{h}(\tau)}-\frac{\hat{\lambda} \beta(1 / \hat{\lambda})}{\dot{h}(\hat{\tau})}\right] d \tau, \quad \hat{\lambda}=\frac{s-\hat{\tau}}{h(\hat{\tau})-x}
$$

where $0<\hat{\tau}<\tau^{*}$. The first term in $(6.1 a)$ will yield the singularity when $\hat{\tau}, \tau^{*}=s$ and $x=$ $h(s)$. However, some care must be used in approaching this limit. Because $\tau^{*}$ lies on $x_{+}$we can write, in view of $(5.5 b)$,

$$
\tau^{*} \sim s+\left(\frac{\partial \tau}{\partial x_{+}}\right) \epsilon, \quad s\left(\frac{\partial \tau}{\partial x_{+}}\right)_{s}=\frac{1}{h(s)-m}<0
$$

where of course $\epsilon=x-h(s)$. Thus, again from $(5.5 b)$ :

$$
x-h\left(\tau^{*}\right) \sim m \frac{x-h(s)}{m-\dot{h}(s)} .
$$

The point $\hat{\tau}$ does not lie on $x_{+}$. Thus $\hat{\tau}=s, x=h(s)$ implies that $\hat{\lambda}=0 / 0($ see $(6.1 b)$ ), and I.'Hospital's rule must be used to show that for $x=h(s)$

$$
\lim _{\hat{\tau} \rightarrow s} \hat{\lambda}=-1 / \dot{h}(s) .
$$

Therefore, as $x \rightarrow h(s)^{+}$the second integral in (5.3) behaves as

$$
-\frac{\beta[\dot{h}(s)]}{\dot{h}^{2}(s)} \ln \left[\frac{m}{h(s)} \frac{x-h(s)}{m-\dot{h}(s)}\right] \text {. }
$$

A similar procedure for $x \rightarrow h(s)^{-}$yields the same result with the signs of $\dot{h}(s)$ and $x-h(s)$

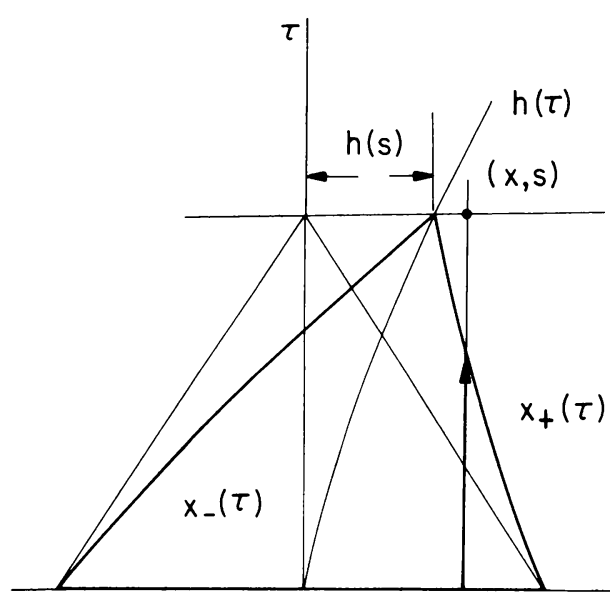

$-m s$
0

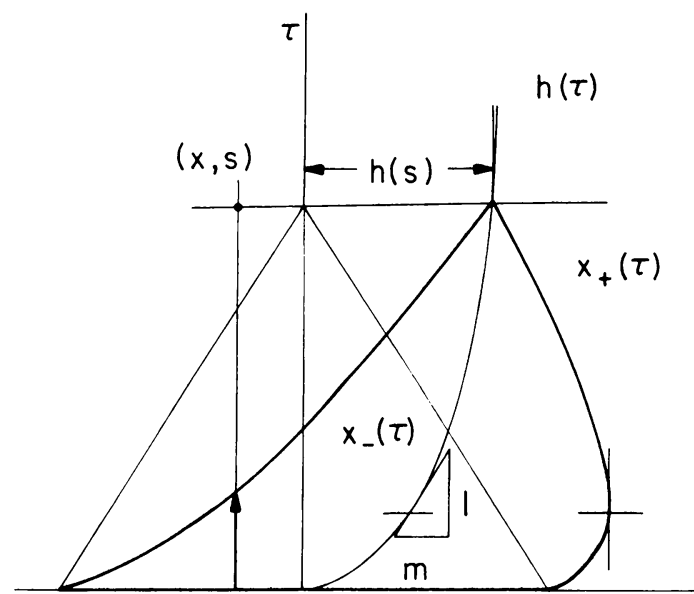

- $\mathrm{ms}$

$\mathrm{ms}$

FIG. 3. Two possible integrations paths for $y=0$. 
reversed. In summary, for $\dot{h}(s)<m$ the non-vanishing stresses and particle velocities on $y$ $=0$ near the wedge edge behave as

$$
\begin{gathered}
\frac{1}{\mu} \sigma_{x} \sim-\frac{m^{2} f^{\prime} M[\dot{h}(s)]}{\pi h^{2}(s) \alpha[\dot{h}(s)]} \ln |x-h(s)|+\cdots, \\
\frac{1}{\mu} \sigma_{y} \sim-\frac{m^{2} f^{\prime} R[\dot{h}(s)]}{\pi h^{2}(s) \alpha[\dot{h}(s)]} \ln |x-h(s)|+\cdots, \\
\dot{u} \sim-\frac{m^{2} f^{\prime} N[\dot{h}(s)]}{\pi \dot{h}(s) \alpha[\dot{h}(s)]} \ln |x-h(s)|+\cdots, \\
M(w)=\left(2-w^{2} / m^{2}\right)\left[2+\left(m^{-2}-2\right) w^{2}\right]-4 \alpha(w) \beta(w), \\
R(w)=4 \alpha(w) \beta(w)-\left(2-w^{2} / m^{2}\right)^{2}, \quad N(w)=2 \alpha(w) \beta(w)+w^{2} / m^{2}-2 .
\end{gathered}
$$

It is readily shown that the $\beta$-components of the non-logarithmic factors in (6.6)-(6.8) vanish when $\dot{h}(s) \geq m$, while the $\alpha$-components behave as $0\left(\epsilon^{-1 / 2}\right), \epsilon \rightarrow 0$ when $\dot{h}(s) \rightarrow 1^{-}$ and vanish for $\dot{h}(s)>1$. Thus, the logarithmic singularities disappear when the wedge edge speed is supersonic. It should also be noted that $M(0), R(0), N(0)=0$ while $M, N>0$ for 0 $<w<m$. However, $R$ is the Rayleigh function and changes from positive to negative as $w$ exceeds $m_{R}=c_{R} / c_{1}$, where $c_{R}<c_{2}$ is the Rayleigh wave speed for the solid. Thus (6.7) indicates that $\sigma_{y}$ at the wedge edge is tensile when the edge speed is below the Rayleigh speed and compressive when that speed is exceeded.

The disturbance arising from the $f(-x)$-term in $(1.2 a)$, i.e. the last two terms in (5.3), do not of course give singularities at the wedge edge. However, it can be shown that at $y=$ $0,|x|=0^{+}$

$$
\frac{1}{\mu} \sigma_{x}, \frac{1}{\mu} \sigma_{y} \sim-\frac{2 f^{\prime}}{\pi}\left(1-m^{2}\right) \ln |x|+\cdots
$$

while $\dot{u}=0$. In the complete problem these singularities would be cancelled by the singular terms existing in the static solution.

7. Brief discussion. The $\sigma_{y}$ generated along $y=0$ is illustrated in Fig. 4 for the case $h$ $=$ constant $<m$, where $m=1 / \sqrt{ } 3$. As indicated in the previous section, $\sigma_{y}$ is tensile at the wedge edge for $\dot{h}<m_{R}$. However, Fig. 4 shows that other regions also exhibit tensile $\sigma_{y}$. The complete problem solution requires superposition of the static stress field. Nevertheless, these results indicate that the normal stress along segments of the wedge surface may be tensile. Physically, the problem in effect requires that adjacent material points in the symmetry plane at the inclusion edge be forced apart. Thus the unbounded tensile stresses at the wedge edge could represent the failure of the solid and be incorporated in a wedge motion criterion. The changing of sign at the Rayleigh wave speed might then imply the existence of a limiting speed, as in brittle fracture theory [5]. However, linear elasticity is violated and perhaps a model of inclusion motion allowing plastic deformation but not singularities near the edge would be more satisfactory. Tensile surface stresses away from the edge may indicate the possibility of wedge-solid separation, i.e. the sundered material does not actually remain in contact with the inclusion surface.

One consideration neglected here is the role of edge sharpness. The present results deal mainly with the wedge. The effect of, for example, a blunt edge, i.e., $f^{\prime}(0) \rightarrow \infty$, was not studied. It should be noted that Freund [6] has treated a mathematically analogous problem of spatially uniform normal stresses on a half-plane and showed that the solution can be applied to the moving dislocation in an unbounded solid. Study of this latter 


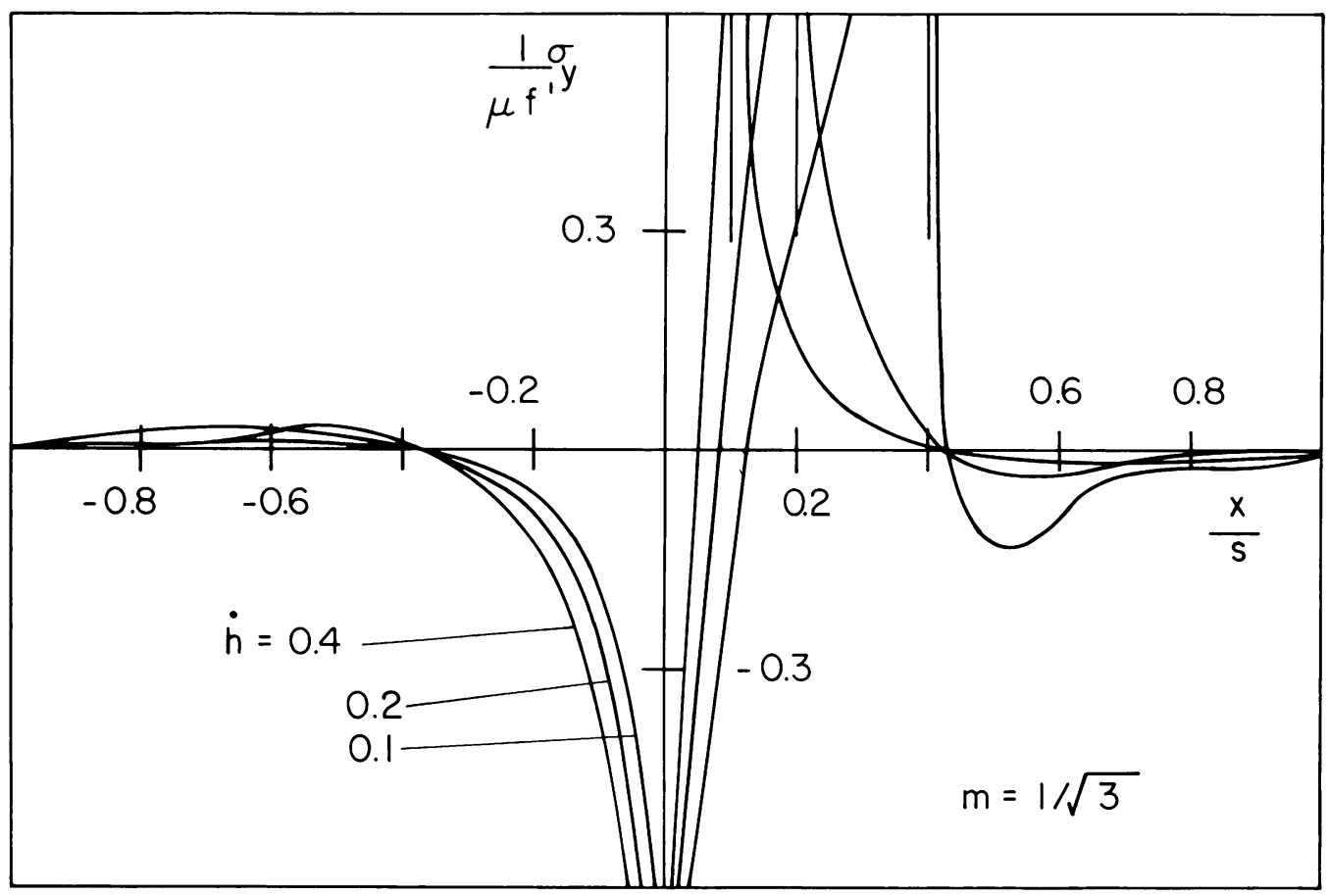

FIG. 4. Normal stress along $y=0$ for wedge at constant subsonic speed,

problem, of course, would yield information on the effect of a blunt-edged inclusion.

This analysis considered smooth inclusions. Thus, the effects of an initial inclusionsolid bond or sliding friction during motion also remain to be studied. One special case, the perfect bond which is broken everywhere along the contact surface at motion initiation, could easily be handled by the present analysis. If the static equilibrium contact shear stress due to the bond is $\sigma(x), x \leq 0$, then $(1.2 b)$ would be replaced by

$$
\sigma_{x y}=-\sigma(x) H(-x) \text {. }
$$

Except for an extra set of terms involving $\sigma$, the solution procedure would be identical. This special case was not treated because $\sigma$ depends on $f$ through the static solution. In this first-step analysis, the wish was to make the results largely independent of this solution, thus preserving, within the limits of travel times for wave reflections, some generality for the loading and geometry in the complete problem. It should be remarked that incorporation of plastic deformation or friction in an analysis might also justify the consideration of thermal effects. In summary, the present analysis is a first step in studying a complicated process. Future work will attempt to incorporate the observations and conjectures made on the basis of this analysis in more physically useful models.

\section{REFERENCES}

[1] N. I. Muskhelishvili, Some basic problems of the mathematical theory of elasticity, Noordhoff International, Leyden, 1975, pp. 492-510

[2] A. T. deHoop, Appl. Sci. Res. B8, 349 (1960)

[3] I. N. Sneddon, The use of integral transforms, McGraw-Hill, New York, 1972, pp. 495-503

[4] I. Stakgold, Boundary value problems of mathematical physics, Vol. I, MacMillan, New York, 1967, pp. 22-24

[5] K. B. Broberg, Ark. Fiz. 18, 159 (1960)

[6] L. B. Freund, J. Appl. Mech. 40, 699 (1973) 